\title{
Thyroid Disorders in Patients with Inflammatory Bowel Diseases
}

\author{
Mustafa Yakut, Yusuf Üstün, Gökhan Kabacan, Irfan Soykan
}

Department of Gastroenterology, Ibni Sina Hospital, Ankara University Medical School, Ankara, Turkey.

Email: isoykan@medicine.ankara.edu.tr

Received November $3^{\text {rd }}, 2010$; revised December $8^{\text {th }}, 2010$; accepted March $14^{\text {th }}, 2011$.

\begin{abstract}
Background: The rate of thyroid disorders is reported to be increased in patients with Ulcerative Colitis (UC) and Crohn's disease $(C D)$ than the normal population. The purpose of this study is to evaluate the prevalance of Thyroid disorders in a group of patients with inflammatory bowel diseases (IBD). Material and Methods: 146 IBD patients (113 UC and 33 CD patients) and 66 healthy control subjects were enrolled into the study. Serum free T4 (FT4), free T3 (FT3), TSH and anti TPO levels of the patients were analyzed retrospectively and and compared with a control group. These cases were also investigated with thyroid ultrasound and nuclear (scintigraphy) imaging. Results: The mean age of IBD patients ( 76 women) was $42.9 \pm 12.4$ years. Among the control group 42 were female and their mean age was $40.9 \pm 12.1$ years. Thyroid gland disease was found in 14 (9.5\%, 8 female, 6 male) of 146 IBD patients. The frequency of thyroid disorders has been found to be higher than the control group in patients with control group (14/146 vs. 1/66, $p=0.042)$. No statistically significant difference has been observed at the thyroid disorder rates between the UC and CD patients $(11 / 113$ vs. $3 / 33, p=0.912)$. Hashimoto thyroiditis has been found at $4(2.7 \%)$ of the IBD patients. Conclusion: In accordance with several works published in literature, we have found a higher rate of thyroid disease apperance at IBD patients.
\end{abstract}

Keywords: Ulcerative Colitis, Crohn's Disease, Thyroid Disorders

\section{Introduction}

Inflammatory bowel diseases (IBD), ulcerative colitis (UC) and Crohn's disease (CD) are diseases that is caused by dysregulation of immune system in patients with genetic predisposition and characterized by chronic inflammation with relapse and remission periods. Ulcerative colitis and $\mathrm{CD}$ are associated with a wide range of extraintestinal complications that influence the course and therapy of the disease. Although extrain-testinal complications of IBD including rheumatologic, ocular, dermatologic and hepatobiliary complications are well understood, the data regarding the relationship between IBD and thyroid disorders are not completely established [1-3]. Epidemiological investigations showed an increased prevalence of hyperthyroidism and IBD. In previous studies, the relationship between UC and thyroid diseases is well established. The rate of thyroid diseases is reported to be higher in UC and CD patients compared to normal population [3-5]. The incidence of thyrotoxicosis is reported as $0.8 \%-3.7 \%$ in UC patients $[2,3,6-8]$. However, the rate of thyroid diseases in $\mathrm{CD}$ patients has been considered in less number of studies than that of UC patients. The aim of this study was to evaluate the prevalance of thyroid disorders in patients with UC and CD.

\section{Material and Methods}

In this retrospective study, a total of 146 patients diagnosed as having inflammatory bowel diseases (113 UC, $33 \mathrm{CD}$ ) were enrolled into the study. All patients had the diagnosis of IBD according to clinical, laboratory, endoscopic and histopathological findings. Medical records of all patients were investigated for their demographic profiles, types of IBD, disease and localisation, and laboratory parameters especially including serum free $\mathrm{T} 4$, free T3, TSH and antiTPO levels. Patients were analyzed retrospectively in terms of age, gender, $\mathrm{UC}$ or $\mathrm{CD}$ localisation. Disease localisation was considered as proctite, left-sided colon involvement, extensive type and pancolitis in UC patients and ileal involvement, colon involvement and ileocolonic involvement in $\mathrm{CD}$ patients. Serum free T4 (FT4), free T3 (FT3), TSH and anti TPO levels of both the patients and the control group were 
analyzed. Patients and control group were diagnosed as having hyperthyroidism, hypothyroidism, subclinical hyperthyroidism, subclinical hypothyroidism and autoimmune thyroiditis based on their thyroid hormone profile and TSH levels, and antithyroid antibody-positivity. These cases were also investigated with thyroid ultrasound and nuclear (scintigraphy) imaging. Serum free T4 (FT4), free T3 (FT3), TSH and anti TPO levels were determined by using radioimmunoassay method. The normal ranges for hormon levels were as follows: TSH: 0.34 - 5.6 micro IU/ml fT4: 7 - 16 pikomol/L, fT3: 7-16 pikomol/L and anti-TPO: 0 - $9 \mathrm{IU} / \mathrm{ml}$.

Data were analyzed with the Statistical Package for Social Sciences (SPSS; version 11.0; SPSS Inc. Chicago, IL) for Windows software. For statistical comparison the Fisher's exact test were used whenever appropriate. Student's t-test was used to compare quantitative variables. Results were expressed a mean $\pm \mathrm{SD}$ and percentages. A value of $p<0.05$ was regarded as significant.

\section{Results}

Patients' demographic characteristics and disease localisation are shown in Tables $\mathbf{1}$ and 2. The mean age of IBD patients was $42.9 \pm 12.4$ years. No statisticaly difference has been observed between the mean ages $(p=$ $0.357)$ and gender distribution $(p=0.136)$ of patients and control group. Patients and control group's thyroid hor- mone profiles and antibody levels are shown in Table 3. When mean fT3 fT4 and TSH levels are taken into consideration, no significant difference was observed between the IBD patients and the control group.

Thyroid gland disease was found in 14 (11 UC, $3 \mathrm{CD})$ of 146 IBD patients. Number and rates of thyroid diseases found in IBD, UC and CD patients were listed in Table 4. The frequency of appearance of thyroid disease has been found to be higher in IBD patients compared to control group (14/146 vs. $1 / 66, p=0.042)$. Five patients had hyperthyroidism and 2 patients had subclinical hyperthyroidism in patients with UC $(5 / 113,4.4 \%$ and 2/113, 1.7\%). As for CD patients, 3 patients were diagnosed as having subclinical hyperthyroidism. No statistically meaningful difference has been observed in the thyroid disorder rates between the $\mathrm{CD}$ and UC patients $(11 / 113$ vs. $3 / 33, p=0.912)$. Hashimoto thyroiditis has been found in $4(2.7 \%)$ of the IBD patients and all of them were UC patients. One of the four patients identified with Hashimoto thyroiditis had hypothyroidism and thyroid hormone treatment was started. Another patient with Hashimoto thyroiditis had rheumatoid arthritis as well. One individual in the control group who was identified as having thyroid disorder had Hashimoto thyroiditis. The frequency of Hashimoto thyroiditis was similar in IBD patients and in the control group $(2.7 \%$ vs. $1.5 \%, p=0.596)$.

Table 1. Baseline characteristics of patients.

\begin{tabular}{ccccc}
\hline Variables & IBD Patients & UC Patients & CD Patients & Control Group \\
\hline$n(\%)$ & $n=146$ & $n=113(77.3 \%)$ & $n=33(22.6 \%)$ & $n=66$ \\
Age (years) & $42.9 \pm 12.4$ & $43.6 \pm 11.5$ & $41.9 \pm 13$, & $40.9 \pm 12.1$ \\
Sex & 70 male & 50 male & 20 male & 24 male \\
& 76 female & 63 female & 13 female & 42 female \\
\hline
\end{tabular}

Table 2. Distribution of the patients.

\begin{tabular}{ccc}
\hline & Crohn's Disease $(n=33)$ & Ulcerative Colitis $(n=113)$ \\
\cline { 2 - 3 } & Terminal Ileum $(62 \%)$ & Distal UC (28.2\%) \\
Location & Colon $(0 \%)$ & Left-sided UC $(44.7 \%)$ \\
& Ileocolon $(38 \%)$ & Extensive UC (12.9\%) \\
Upper GI $(0 \%)$ & Pancolitis $(14.1 \%)$ \\
\hline
\end{tabular}

Table 3. Patients and control group's thyroid hormone profiles and antithyroid antibody levels.

\begin{tabular}{lcccc}
\hline & Free T3 (picomol/L) & Free T4 (picomol/L) & TSH (micro IU/ml) & Anti TPO (IU/ml) \\
\hline IBD & $4.4 \pm 1.27$ & $14.4 \pm 4.01$ & $2.1 \pm 3.6$ & $14.1 \pm 29.1$ \\
UC & $4.5 \pm 1.42$ & $14.3 \pm 4.015$ & $1.8 \pm 2.13$ & $14.2 \pm 30.25$ \\
CD & $4.3 \pm 0.89$ & $14.46 \pm 3.81$ & $2.7 \pm 5.59$ & $14.07 \pm 28.35$ \\
Control Group & $4.8 \pm 0.85$ & $15.04 \pm 2.9$ & $4 \pm 15.3$ & $22.4 \pm 82.6$ \\
\hline
\end{tabular}


Table 4. The rate of thyroid disease in IBD patients.

\begin{tabular}{lcccc}
\hline & IBD $(n=146)$ & UC $(n=113)$ & CD $(n=33)$ & Control Group $(n=66)$ \\
\hline Thyroid Disease & $14(9.5 \%)$ & $11(9.7 \%)$ & $3(9.0 \%)$ & $1(1.5 \%)$ \\
Hyperthyroidism & $5(3.4 \%)$ & $5(4.4 \%)$ & $0(0 \%)$ & $0(0 \%)$ \\
Hypothyroidism & $1(0.06 \%)^{*}$ & $0(0 \%)$ & $0(0 \%)$ & $0(0 \%)$ \\
Subclinical Hypothyroidism & $0(0 \%)$ & $0(0 \%)$ & $0(0 \%)$ & $0(0 \%)$ \\
Subclinical Hyperthyroidism & $5(3.4 \%)$ & $2(1.7 \%)$ & $3(9.0 \%)$ & $0(0 \%)$ \\
Thyroidits & $4(2.7 \%)$ & $4(3.5 \%)$ & $0(0 \%)$ & $1(1.5 \%)$ \\
\hline
\end{tabular}

\section{Discussion}

In the present study, 146 patients with IBD, among which 113 were UC and 33 were $\mathrm{CD}$, were evaluated. It has been observed that thyroid disease occurance rate is higher than that of the control group (9.5\% vs $1.5 \%)$. Thyroid disorders were observed in $9.7 \%$ of the patients with UC and in $9.0 \%$ of the patients with CD. The incidence of hyperthyroidism and subclinical hyperthyroidism was found to be higher in IBD patients compared to the control group. However, hypothyroidism and thyroiditis frequencies were similar to that of the control group $(2.7 \%$ vs. $1.5 \%, p=0.596)$. However, no significant difference has been observed in patients with UC compared to patients with CD patients.

In some studies, occurance of thyroid diseorders in IBD patients is reported to be at similar frequencies compared to normal population. In a population based study performed in Canada in which 8,072 IBD patients were evaluated, thyroiditis rate was $0.23 \%$ in UC patients and $0.19 \%$ in CD patients (versus $0.15 \%-0.20 \%$ in controls). In this study thyroid disease frequency is found to be similar to that of normal population [9]. In a study performed in our country in which 25 ulcerative colitis patients were evaluated, thyroid disease frequency is reported to be similar to that of control group [10]. In a study performed in Italy, in which $162 \mathrm{UC}$ patients were evaluated thyroid disorders (hyper-and hypothyroidism) are reported at the rate of $2.5 \%$. In the same geographic region, the rate has been reported as $7.5 \%$ in normal population [6]. In a recent study performed in Italy in which 909 IBD patients are evaluated thyroid disorder rates are reported as $6.6 \%$ in all IBD patients, $6.89 \%$ in $\mathrm{CD}$ patients and $6.29 \%$ in UC patients. In this study Hashimoto thyroidis rates are reported as $1.98 \%$ in all IBD patients, $2.15 \%$ in $\mathrm{CD}$ patients and $1.8 \%$ in UC patients. As a result of this study it has been concluded that thyroid disorder rate is twice in IBD patients than that in healthy European population [3]. Subclinical hyperthyroidism also has been reported in patients with in ulcerative colitis [11]. Though in most studies thyroid disorders are associated with only UC, in a limited number of cases thyroid diseas has been reported also in patients with $C D$ $[12,13]$. In some studies tT4 level is reported to decrase in CD patients. Also in these studies it has been shown that frequency of antithiroglobulin and antithyroid mikrosomal/peroxidase antibodies increases in IBD patients $[13,14]$. It is reported that making laboratory tests for hyperthyroidism in patients with ulcerative co- litis is necessery $[15,16]$.

Some of the advantages and shortcomings of this study can be listed as follows: in the present study CD patients, which are studied less in the literature, as well are evaluated together with UC patients. The rates of thyroid diseases observed in IBD patients are classified according to thyroid disease groups (thyroiditis, hyper- thyroidism, hypothyroidism, subclinical hyperthyroidism, subclinical hypothyroidism). Number of patients is large enough in this field and comparisons are made with a control group. The weakness of the study is that, this was a retrospective study and population based evaluation has not been performed.

In conclusion, although the thyroid disease prevalance in IBD patients is reported to be similar to that in normal population in some studies, we have found an increased rate of thyroid diseases in IBD patients in accordance with most of the studies. It is necessary to make laboratory tests for hyperthyroidism in patients with ulcerative colitis in which treatment-resistant and/or enlarged thyroid gland is observed. Early diagnosis of thyroid diseases is important because thyroid diseases may worsen the course and symptoms of IBH.

\section{REFERENCES}

[1] J. Powell, H. Shapiro and J. Carbone, "Therappeutic Problems of Ulcerative Colitis with Hyperthyroidisim," American Journal of Gastroenterology, Vol. 50, No. 2, 1968, pp. 116-124.

[2] G. Jarnerot, A. K. Azad Khan and S. C. Truelove, "The Thyroid in Ulcerativecolitis and Crohn's Disease. II. Thyroid Enlargement and Hyperthyroidism in Ulcerative 
Colitis," Acta Medica Scandinavica, Vol. 197, 1975, pp. 83-87. doi:10.1111/j.0954-6820.1975.tb04882.x

[3] M. Cesarini, E. Angelucci and M. Rivera, "Thyroid Disorders and Inflammatory Bowel Diseases: Retrospective Evaluation of 909 Patients from an Italian Referral Center," Inflammatory Bowel Diseases, Vol. 16, No. 2, 2010, pp. 186-187.

[4] A. J. Greenstein, H. D. Janowitz and D. B. Sachar, "The Extra-Intestinal Complications of Crohn's Disease and Ulcera-Tivecolitis a Study of 700 Patients," Medicine, Vol. 55, No. 5, 1976, pp. 401-412. doi:10.1097/00005792-197609000-00004

[5] P. Gimondo, P. Mirk, C. Pizzi, G. Messina and S. Gimondo, "Iafrancesco G. Clinico-Ultrasonographic Assessment of the Thyroid Volume and Function in Chronic Enteritis and Colitis: Preliminary Data," Radiologia Medica, Vol. 92, No. 3, 1996, pp. 257-260.

[6] G. Casella, E. De Marco, E. Antonelli, et al., "The Prevalence of Hyper and Hypothyroidism in Patients with Ulcerative Colitis," Journal of Crohn's and Colitis, Vol. 2, No. 4, 2008, pp. 327-330. doi:10.1016/i.crohns.2008.09.001

[7] D. Peretianu, C. Dumitrescu and V. Lotrenau, "Association of Hyperthyroidism and Ulcerative Colitis. Report of Two Cases," Medicine Interne, Vol. 28, No. 1, 1990, pp. 41-45.

[8] J. K. Triantafillidis, P. Cherakakis and A. Zervakakis "Coexistence of Hyperthyroidism and Ulcerative Colitis: Report of 4 Cases and a Review of the Literature," Italian Journal of Gastroenterology, Vol. 24, No. 9, 1992, pp. 494-497.

[9] C. N. Bernstein, A. Wajda and J. F. Blanchard, "The Clustering of Other Chronic Inflammatory Diseases in Inflammatory Bowel Disease: A Population-Based Study,"
Gastroenterology, Vol. 129, 2005, pp. 827-836 doi:10.1053/j.gastro.2005.06.021

[10] B. Kantarçeken, A. Çetinkaya and M. Şahin, "Thyroid Hormon Levels in Ulcerative Colitis Patients," Akademik Gastroenteroloji Dergisi, Vol. 77, No. 33, 2008, pp. 168170.

[11] B. Tunc, L. Filik, A. Ulker, A. Demirbag and B. Sahin "Subclinical Thyroid Disorders and Inflammatory Bowel Disease," Romanian Journal of Gastroenterology, Vol. 14, No. 1, 2005, pp. 98-99.

[12] S. A. Shah, M. A. Peppercorn and J. A. Pallotta, "Autoimmune (Hashimoto's) Thyroiditis Associated with Crohn's Disease," Journal of Clinical Gastroenterology, Vol. 26, No. 2, 1998, pp. 117-120. doi:10.1097/00004836-199803000-00006

[13] I. Bank and J. O. Busari, "Crohn's Disease, Autoimmune Thyroiditis, and Beta-Thalassemia Trait in an Adolescent: An Unusual Combination of Diseases," European Journal of Pediatrics, Vol. 167, No. 11, 2008, pp. 1343-1346. doi:10.1007/s00431-008-0676-3

[14] G. Messina, N. Viceconti and B. Trinti, "The Clinical and Echographic Assessment of Thyroid Function and Structure in Patients with a Chronic Inflammatory Intestinal Disease," Recenti Progressi in Medicina, Vol. 90, No. 1, 1999, pp. 13-16.

[15] X. Geng, L. Biancone and H. H. Dai, et al. "Tropomyosin Isoforms in İntestinal Mucosa: Production of AutoantiBodies to Tropomyosin Isoforms in Ulcerative Colitis," Gastroenterology, 1998, Vol. 114, No. 5, pp. 912-922. doi:10.1016/S0016-5085(98)70310-5

[16] H. Hasegawa, K. Hasegawa, T. Saito, et al., "A Case of Myopathy with Ulceratif Colitis," Clinical Neurology, Vol. 30, No. 2, 1990, pp. 184-188. 